\title{
FRONTO-STRIATAL COGNITIVE DEFICITS AT DIFFERENT STAGES OF PARKINSON'S DISEASE
}

\author{
by A. M. OWEN, ${ }^{1}$ M. JAMES, ${ }^{2}$ P. N. LEIGH, ${ }^{3}$ B. A. SUMMERS, ${ }^{3}$ \\ C. D. MARSDEN, ${ }^{4}$ N. P. QUINN, ${ }^{4} \mathrm{~K}$. W. LANGE ${ }^{4}$ and T. W. ROBBINS \\ (From the 'Department of Experimental Psychology, University of Cambridge, the ${ }^{2}$ Department of \\ Psychology, The National Hospital for Neurology and Neurosurgery, the ${ }^{3}$ Department of Neurology, \\ Institute of Psychiatry, and the ${ }^{4}$ Department of Clinical Neurology, Institute of Neurology, \\ London, UK)
}

\begin{abstract}
SUMMARY
Groups of patients with idiopathic Parkinson's disease, either medicated or unmedicated, were compared with matched groups of normal controls on a computerized battery previously shown to be sensitive to frontal lobe dysfunction, including tests of planning, spatial working memory and attentional set-shifting. In a series of problems based on the 'Tower of London' test, medicated patients with Parkinson's disease were shown to be impaired in the amount of time spent thinking about (planning) the solution to each problem. Additionally, an impairment in terms of the accuracy of the solution produced on this test was only evident in those patients with more severe clinical symptoms and was accompanied by deficits in an associated test of spatial short-term memory. Medicated patients with both mild and severe clinical symptoms were also impaired on a related test of spatial working memory. In contrast, a group of patients who were unmedicated and 'early in the course' of the disease were unimpaired in all three of these tests. However, all three Parkinson's disease groups were impaired in the test of attentional set-shifting ability, although unimpaired in a test of pattern recognition which is insensitive to frontal lobe damage.

These data are compared with those previously published from a group of young neurosurgical patients with localized excisions of the frontal lobes and are discussed in terms of the specific nature of the cognitive deficit at different stages of Parkinson's disease.
\end{abstract}

\section{INTRODUCTION}

Many studies have now established that patients with Parkinson's disease develop mild neuropsychological deficits across a range of cognitive functions (Lees and Smith, 1983; Boller et al., 1984; Weingartner et al., 1984; Pillon et al., 1986; Taylor et al., 1986; Sagar et al., 1988a; Ogden et al., 1990; see also a review by Brown and Marsden, 1988). Although some of these impairments closely resemble those commonly attributed to frontal lobe dysfunction, in general direct comparisons have not been made between patients with Parkinson's disease and patients with frontal lobe damage.

One exception is in the area of attentional set formation and set shifting. Several studies have shown that frontal lobe patients perform poorly on the Wisconsin Card Sorting Test, achieving fewer sorting categories and making more perseverative errors (Milner, 1964). Evidence for a frontal-like set-shifting impairment has also been reported for both medicated (Bowen et al., 1975; Taylor et al., 1986; Canavan et al., 1989) and non-medicated patients with Parkinson's disease (Lees and Smith, 1983; Canavan et al., 1989), although the deficits are generally much smaller than those associated with

Correspondence to: Dr A. M. Owen, Department of Experimental Psychology, Downing Street, Cambridge CB2 3EB, UK. 
frontal lobe lesions (see Brown and Marsden, 1988). The precise cognitive processes responsible for impaired Wisconsin Card Sorting Test performance in Parkinson's disease have been investigated using a number of associated attentional set-shifting paradigms (Cools et al., 1984; Flowers and Robertson, 1985; Downes et al., 1989). In the recent study by Downes et al. (1989) both medicated and non-medicated patients with Parkinson's disease were impaired on a test of visual discrimination learning in which subjects were required to shift response set between two stimulus dimensions. In a parallel study, a young group of neurosurgical patients with localized excisions of the frontal lobes were also shown to be impaired on this task, although no deficits were found in groups of patients with unilateral temporal lobe or amygdala-hippocampus excisions (Owen et al., 1991).

Patients with frontal lobe damage are often described as lacking normal executive control over action, as exemplified by their deficits in the cognitive aspects of planning on the Tower of London test (Shallice, 1982). Using a computerized version of this test, patients with frontal lobe excisions have recently been shown to have a characteristic profile of impairment in terms of both the accuracy and the latency of thinking (Owen et al., 1990). They required more moves to complete these planning problems and a 'yoked' motor control condition has revealed that movement times were significantly increased in this group. Taking both of these factors into consideration, initial thinking (planning) time was unimpaired in the frontal lobe group, although the amount of time spent thinking subsequent to the first move was significantly prolonged.

Taylor et al. (1986) have suggested that parkinsonian patients are impaired in tasks that involve 'self-directed behavioural planning', although in a later study no deficits were found using a three disc planning problem similar in design to the Tower of London test (Saint-Cyr et al., 1988). In a preliminary study, we investigated planning performance in patients with Parkinson's disease using the computerized Tower of London test (Morris et al., 1988). A group of 12 medicated patients with mild or moderately severe clinical symptoms was no less accurate than controls in solving the Tower of London planning problems but spent a significantly greater amount of time thinking about the solution prior to (but not subsequent to) making the first move, a pattern of deficits that contrasted with the frontal lobe patients described by Owen et al. (1990).

There are, however, several problems of comparison between these studies of frontal lobe patients (Owen et al., 1990) and patients with idiopathic Parkinson's disease (Morris et al., 1988). For example, whilst the Tower of London test used in the two studies was conceptually similar, there were radical differences in the actual appearance and presentation of the planning problems that may have contributed to the different performance of the two patient groups. In addition, the methods used to calculate estimates of initial and subsequent 'thinking' time unconfounded by movement times were refined in the later study (Owen et al., 1990). Specifically, the estimates of movement time needed to make these corrections were derived directly from the actual sequence of moves carried out by the subject, rather than from the 'ideal' or correct sequence as defined by the problem itself.

The deficits in the Tower of London test in patients with frontal lobe damage and Parkinson's disease have been considered in terms of the component processes required for efficient planning. One obvious possibility is that the set-shifting impairments seen in these two groups may contribute to the deficits in planning, given the importance 
of 'set' for efficient problem solving (see Gibson, 1941). Another possibility is that the deficits reflect an impairment of memory function. The Tower of London test requires an active search of possible solutions, placing a significant load on spatial working memory. Subsequently, the problem solution must be held in spatial short-term memory and transposed into the appropriate sequence of motor movements before it can actually be executed. In the two studies described in detail above (Morris et al., 1988; Owen et al., 1990) specific tests were employed to assess the individual efficiency of some of these component processes. Spatial short-term memory span was assessed using a paradigm based on Corsi's block-tapping task (Milner, 1971), which provided a simple index of the subject's ability to retain, transpose and finally execute a defined sequence of spatial moves. A self-ordered search task was also employed to measure the efficiency of spatial working memory which has been reported to be impaired in individuals with frontal lobe pathology (Petrides and Milner, 1982).

The test of spatial short-term memory revealed no significant impairment in the frontal lobe patients' ability to execute a sequence of visuo-spatial moves. In contrast, the spatial working memory paradigm revealed significant impairments in this patient group in both possible types of search error and this was related to the inefficient use of a particular search strategy for solving the problems (Owen et al., 1990). No impairments were reported in patients with Parkinson's disease using similar but not identical tests of spatial span and spatial working memory (Morris et al., 1988).

There are further problems of comparison between these two studies of patients with frontal lobe damage and patients with idiopathic Parkinson's disease arising from the progressive nature of Parkinson's disease and the possibility that the cognitive deficits are quantitatively and qualitatively different at various stages of the disease. In recent years several studies have emphasized the need to take account of the severity of clinical symptoms when assessing cognitive impairments in Parkinson's disease. For example, Mortimer et al. (1982) found a relationship between the degree of bradykinesia and visuo-spatial deficit, whilst Taylor et al. (1986) found correlations between the severity of the clinical (physical) symptoms and the performance on several cognitive tasks. It should be emphasized, however, that if such a relationship exists, it is not simply a direct one between impaired motor and cognitive function, since many patients can fluctuate between extremes of motor disability whilst aspects of cognitive function remain unaffected (Brown et al., 1984). Furthermore, several studies have found insignificant or weak associations between measures of cognitive and motor performance (Sahakian et al., 1988; Ogden et al., 1990).

Medication may also play a key role in the cognitive performance of parkinsonian patients. Several studies have reported improved function in certain aspects of frontal lobe test performance when L-dopa is administered (Bowen et al., 1975), whilst in others the reverse pattern has been found (Gotham $e t a l$., 1988). The likely role of medication in cognition can be assessed by studying $\mathrm{F}$ atients who are 'early-in-the course' of Parkinson's disease and are yet to receive any medication. Using this approach, several studies have now reported specific cognitive deficits in such groups of patients (Lees and Smith, 1983; Taylor et al., 1987; Downes et al., 1989). In the study of Downes et al. (1989), the deficits in set-shifting ability were, if anything, worse in the 'earlyin-the-course' unmedicated patients than in the medicated patients with more severe clinical symptoms. 
In the present investigation, the computerized Tower of London test, and tests of spatial working memory and spatial span, were used to study planning in three subgroups of patients with idiopathic Parkinson's disease. An initial group of 44 patients was divided into those who were non-medicated with mild clinical symptoms, those that were medicated with mild or moderate clinical symptoms and those who were medicated with more severe clinical symptoms. By using precisely the same battery of tests adopted by Owen et al. (1990) to investigate patients with frontal lobe damage, direct comparisons could be made with this earlier study and, in addition, the likely contribution of both medication and the severity of clinical symptoms in Parkinson's disease could be assessed.

A test of pattern recognition (Sahakian et al., 1988) was also given to assess the psychological specificity of any effects observed. This test is not sensitive to localized excisions of the frontal lobes (A. M. Owen, unpublished results), although impairments have been observed in patients with both mild and moderate dementia of the Alzheimer type (Sahakian et al., 1988; Sahgal et al., 1991).

Although both medicated and non-medicated patients with Parkinson's disease have previously been shown to be impaired on a test requiring a shift of attentional set to a previously irrelevant dimension (Downes et al., 1989), all three patient groups assessed in the present study were also given an identical version of this test in order that direct comparisons might be made between these patients and those used in the earlier study.

\section{METHODS}

\section{Subjects}

The 44 parkinsonian patients included in this study were all outpatients at the Maudsley Hospital and the National Hospital for Neurology and Neurosurgery, London. In all cases, idiopathic Parkinson's disease was diagnosed by a consultant neurologist who also assessed the severity of clinical symptoms according to the Hoehn and Yahr rating scale (Hoehn and Yahr, 1967). In cases where patients were experiencing response fluctuations the Hoehn and Yahr rating referred to the 'on' rather than the 'off' condition. On the basis of this assessment, and depending on whether anti-parkinsonian medication had already been received, each patient was assigned to one of three groups.

Fifteen patients were early in the course of the disease (mean $=18 \mathrm{mths}$ ) and had not yet received any medication (non-medicated Parkinson's disease). In this group, clinical symptoms were rated either as Hoehn and Yahr stage I (three patients), stage II (10 patients) or stage III (two patients).

The remaining 29 patients were all receiving $L$-dopa preparations either alone or in combination with other medication. Fifteen of these individuals had mild/moderate physical symptoms (medicated Parkinson's disease (mild)] and were rated as either Hoehn and Yahr stage I (three patients) or stage II (12 patients). In addition to their dopaminergic treatment, four of these patients were receiving anti-cholinergic medication (orphenadrine or benzhexol) at the time of testing. The mean daily dose of L-dopa was $575 \mathrm{mg}$.

The remaining 14 patients [medicated Parkinson's disease (severe)] had more severe physical symptoms and were rated as stage III (eight patients) or stage IV (six patients). Three of these patients were receiving anticholinergic as well as dopaminergic medication at the time of testing (orphenadrine or benzhexol). The mean daily dose of L-dopa for this group was $779 \mathrm{mg}$.

Exclusion criteria for the two groups of medicated Parkinson's disease patients included clinical dementia assessed using both the Mini Mental State Examination (MMSE) (Folstein et al., 1975) and the Kendrick Object Learning Test (KOLT) (Kendrick, 1985). Specifically, this study only included patients who scored 24 out of 30 or above on the MMSE and 23 points or above on the KOLT.

To assess the incidence of affective disturbance in these patients, the Geriatric Depression Scale (GDS) (Yesavage et al., 1982) was also administered. This is a self-administered, 30-item questionnaire designed specifically for elderly subjects. It is particularly suited for the assessment of depression in parkinsonian patients since it contains relatively few somatic items which may relate directly to the patients' physical disability. In the present study, data from the GDS were analysed twice, including and excluding somatic 
items. Since no qualitative difference was found between the two analyses, only the results from the initial, fully inclusive analysis are presented. The non-medicated patients were not screened for dementia or depression. Informed consent was obtained from all patients.

Three groups of healthy control subjects $(n=44)$ were chosen to match the groups of parkinsonian patients as closely as possible with respect to age and pre-morbid verbal IQ as assessed using the National Adult Reading Test (NART) (Nelson, 1982). These subjects were drawn from a large pool of control volunteers at the North East Age Research panel in Newcastle upon Tyne, with informed consent.

Table 1 shows a summary of characteristics for the three patient groups and their controls. One-way analysis of variance (ANOVA) confirmed that the non-medicated Parkinson's disease group, the nonmedicated Parkinson's disease (mild) group and the medicated Parkinson's disease (severe) group were all well matched with their respective control groups in terms of age $[\mathrm{F}(1,28)=0.66, \mathrm{~F}(1,28)=0.13$, $F(1,26)=0.59$, respectively] and NART IQ estimate $[F(1,28)=1.62, F(1,28)=1.67, F(1,26)=1.71$, respectively]. The two medicated groups did not differ significantly in terms of their MMSE scores $[\mathrm{F}(1,22)$ $=0.31]$ or their performance on the $\operatorname{KOLT}[F(1,24)=3.3]$. There was, however, a significant difference between these two groups in their scores on the $\operatorname{GDS}[\mathrm{F}(1,25)=8.11, P<0.01]$ and this will be discussed in detail later. In addition, a Newman-Keuls comparison between the three patient groups revealed that the medicated Parkinson's disease (mild) and the medicated Parkinson's disease (severe) subgroups both differed significantly from the non-medicated Parkinson's disease group in terms of disease duration $[F(2,39)=4.25, P<0.025]$, although the two medicated groups did not differ significantly from each other in this measure.

In the attentional set-shifting task, the three groups of Parkinson's disease patients were compared with a large group of normal control volunteers $(n=129$, mean age $=57.15 \mathrm{yrs}$, mean NART IQ $=114.7)$ drawn from the North East Age Research Panel in Newcastle upon Tyne and from the Newcastle, Cambridgeshire and London areas. Since this part of the study was a replication of an earlier investigation (Downes et al., 1989), included for the purpose of comparison, the set-shifting task was administered to a subset of the parkinsonian patients, in a separate test session. In all, 14 non-medicated Parkinson's disease patients and nine from each of the medicated Parkinson's disease groups were tested.

\section{Materials and procedure}

The main testing procedures were taken from the Cambridge Neuropsychological Test Automated Battery, a series of computerized paradigms run on an Acorn BBC Master microcomputer with a high resolution Microvitec colour monitor and a Microvitec (Touchtech 501) touch sensitive screen. Subjects were seated approximately $0.5 \mathrm{~m}$ from the monitor and it was explained that they would have to respond to stimuli by touching the screen. They were introduced to the apparatus by way of a 'motor screening task' in which they were asked to respond to a series of flashing crosses on the screen by placing the index finger of their preferred hand on the centre point of each cross. Once each cross had been accurately touched the next cross appeared after a brief delay. Following a short demonstration by the experimenter, in which three consecutive crosses were touched, subjects were presented with a series of 10 crosses to touch at $6 \mathrm{~s}$ intervals. After satisfactorily completing the introductory motor screening task, subjects were given the following three tests in the order described below.

TABLE I. SUBJECT CHARACTERISTICS

\begin{tabular}{|c|c|c|c|c|c|c|c|c|c|}
\hline Group & $\begin{array}{l}\text { Stage } \\
{[\text { mean }]}\end{array}$ & $n$ & Age (yrs) & NART & $\begin{array}{c}\text { Disease } \\
\text { duration } \\
\text { (yrs) }\end{array}$ & $\begin{array}{l}\text { L-dopa } \\
\text { (mg) }\end{array}$ & MMSE & GDS & KOLT \\
\hline $\begin{array}{l}\text { Non-medicated Parkinson's } \\
\text { disease }\end{array}$ & $1-\mathrm{IJ}[[1.93]$ & 15 & $55.73(2.9)$ & $111.8(2.5)$ & $1.5(4.2)$ & & & & \\
\hline Controls & & 15 & $58.33(1.49)$ & $115.7(1.6)$ & & & & & \\
\hline $\begin{array}{l}\text { Medicated Parkinson's } \\
\text { disease (mild) }\end{array}$ & I-II[1.8] & 15 & $58.86(2.83)$ & $108(2.79)$ & $7.07(1.27)$ & $575(248)$ & $28.25(0.57)$ & $8.61(1.78)$ & $38.16(2.1)$ \\
\hline Controls & & 15 & $60.06(1.84)$ & $112(1.5)$ & & & & & \\
\hline $\begin{array}{l}\text { Medicated Parkinson's } \\
\text { disease (severe) }\end{array}$ & $\mathrm{III}-\mathrm{IV}[3.43]$ & 14 & $65.85(1.73)$ & $107.9(2.56)$ & $10.21(1.57)$ & $779(273)$ & $27.83(0.52)$ & $15.14(1.55)$ & $33.78(1.46)$ \\
\hline Controls & & 14 & $64.14(1.26)$ & $112(1.7)$ & & & & & \\
\hline
\end{tabular}

Standard errors are shown in round brackets, NART = National Adult Reading Test: MMSE = Mini Mental State Examination; GDS = Geriatric Depression Scale: KOLT $=$ Kendrick Object Learning Test. 


\section{Spatial short-term memory task (Fig. 1A)}

In this computerized version of the Corsi's block-tapping task (Milner, 1971), spatial short-term memory capacity was determined from the ability of subjects to remember a sequence of squares on the screen. Each trial began with the same arrangement of nine white $3 \mathrm{~cm}$ squares, presented on the screen in a pseudorandom pattern. Subjects were instructed to observe the boxes, as some would change colour one after the other. Their task was to remember the location and the sequential order of the boxes which changed. During each series, one box would change colour for $3 \mathrm{~s}$, and then return to white before the next in the sequence changed to the same colour. The subject was then prompted by a tone to repeat the sequence by touching the boxes in the same order. During this response sequence, each selected box changed to the same colour for $1 \mathrm{~s}$, and a feedback tone sounded. Following one demonstration trial by the experimenter, the task began at the simplest level of difficulty with a two box sequence. After each successful trial, the number of boxes changing in the next sequence was increased by one to a maximum of nine boxes. After an incorrect attempt at any particular level, an alternative sequence of the same length was presented. This continued until the subject had failed three consecutive trials at any one level. During each trial, a number in the bottom left-hand corner of the screen indicated the length of the current sequence. Also, all boxes changed to the same colour within each series, although on any two adjacent sequences different colours were used to minimize interference. The spatial short-term memory span was calculated as the highest level at which the subject had successfully recalled at least one sequence of boxes.

\section{Spatial working memory task (Fig. 1B)}

In this task the subject was required to 'search through' a number of boxes presented on the screen by touching each one such that it 'opened up', revealing what was inside. The objective was to collect 'blue tokens' hidden inside the boxes and, once found, to use them to fill an empty column at the side of the screen. The subjects were instructed that at any one time there would be a single token hidden inside one of the boxes. Their task was to search until they found it, at which point the next token would be
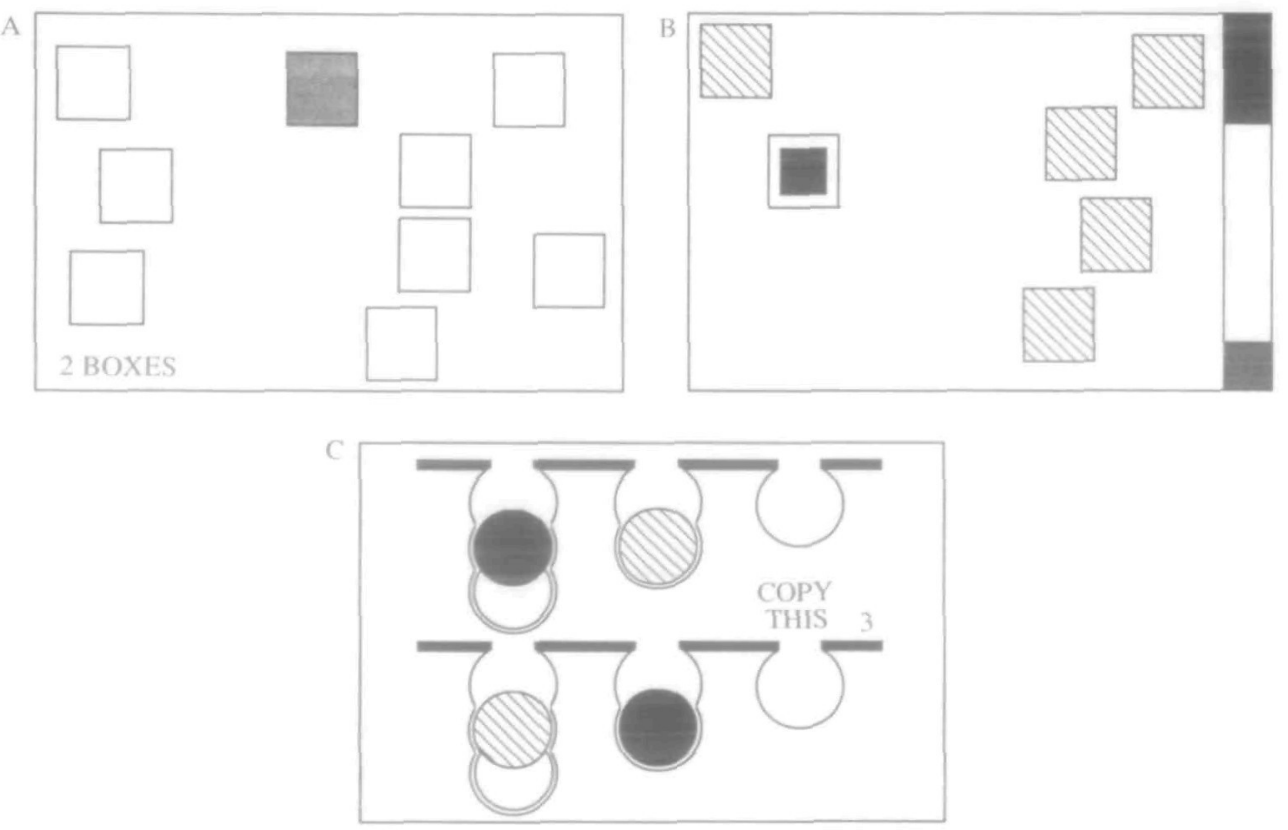

FIG. 1. A, the spatial span test. B, the spatial working memory test (a six box problem is shown). c. a sub-problem from the computerized Tower of London test. Three moves (indicated) are required to make the two arrangements identical. 
hidden. The key instruction was that once a blue token had been found within a particular box, then that box would never be used again to hide a token. Since every box was used once, on every trial the total number of blue tokens to be found corresponded to the number of boxes on the screen. In this task, two types of search error were possible. First, a subject may return to open a box in which a blue counter has already been found (a 'between search error'). Secondly, a subject may return to a box already opened and shown to be empty earlier in the same search (a 'within search error'). Subjects could search the boxes in any order but, for control purposes, the number of empty boxes visited (excluding errors) before a token was found was determined by the computer. Thus, each subject received the same degree of feedback prior to the first error. After four practice trials with three boxes, there were four test trials with each of four, six and finally eight, boxes. The task was scored according to the number of between- and withinsearch errors at each level of difficulty. One possible strategy for completing this task is to follow a predetermined search sequence, beginning with a particular box and then returning to start each new sequence with that same box as soon as a token has been found. A previous investigation has shown that impaired performance of this task may be related to inefficient use of this particular search strategy in neurosurgical patients with frontal lobe excisions (Owen et al., 1990). In this study, the extent to which parkinsonian patients and their controls used this repetitive searching pattern as a strategy for approaching the problem was estimated from the number of search sequences starting with the same box, within each of the more difficult six and eight box problems. The total of these scores provided a single measure of strategy for each subject, with a high score (many sequences beginning with a different box) representing low use of the strategy and a low score (many sequences starting with the same box) representing more extensive usage.

\section{Planning task (Fig. 1C)}

This task is a variation on one developed by Shallice and McCarthy (Shallice, 1982) based on the 'Tower of Hanoi' problem. Two sets of three coloured balls were presented, one in the top half of the screen and one in the bottom half. These were described to the subject as snooker balls since they appeared to be hanging in 'pockets' or 'socks'. There were three pockets in each half of the screen, one that could clearly hold three balls, one that could hold two balls, and one that would be completely filled by just one ball. On each trial a red ball, a blue ball and a green ball were placed in predetermined positions in the pockets of each of the two displays. The subject was asked to rearrange the balls in the bottom display, such that their positions matched the 'goal' arrangement in the top half of the screen. A ball could be moved by first touching it and then by touching an empty position in one of the other pockets. Once selected, a tone sounded and the rim of the ball began to flash, indicating that it was ready to be moved. At any time, the subject could cancel a selected ball by touching it a second time. 'Illegal' moves, such as trying to place a ball high in a pocket when there was no other ball beneath it, or trying to remove a ball while there was another sitting above it in the same pocket, were carefully explained to the subject and if attempted, they were registered, but evoked no response from the computer.

The starting position of the balls was varied such that in any particular trial the solution could only be reached after a minimum of two, three, four or five moves. Subjects were instructed to examine the position of the balls at the beginning of each problem and attempt to solve it in the minimum possible number of moves. This was given to them verbally and displayed on the screen throughout each trial. They were encouraged not to make the first move until they were confident that they could execute the entire sequence needed to solve the problem. The maximum moves allowed corresponded to twice the minimum number possible plus one, or plus two in the case of 'five-move' problems. Following successful completion of each problem, the computer gave the word 'FINISHED', before moving onto the next trial. If the maximum number of moves was reached, 'TOO MANY MOVES' appeared before the beginning of the next trial. The program stored the number of moves required by the subject to rearrange the balls and measured the selection and movement latencies for both the first and subsequent moves. After six practice problems with one and two moves, the subject was given two each of two- and three-move problems and four each of four- and five-move problems. These test problems corresponded exactly to those used in the original Tower of London test (Shallice, 1982).

For each test problem, a 'yoked control' condition was employed to provide baseline measures of motor initiation and execution times. On each trial of this control condition, the subject was required to follow a sequence of single moves executed by the computer in the top half of the screen by moving the corresponding ball in the lower arrangement. Thus, initially the two arrangements differed by just one ball. Once the 
subject had made the appropriate move, the top arrangement changed again so the subject had to make another single move. The test was 'yoked' to the main test in the sense that in each trial, the movement of balls was an exact replication of those moved by the subject in the corresponding test trial. The measurement of selection and execution latencies in this control condition provided baseline estimates of motor initiation and execution times.

Test trials and 'yoked control' trials were arranged in four blocks of six problems each. The first six test trials were given (two problems at each of two, three and four moves), followed by their corresponding 'yoked control' trials. Then the remaining six test problems were presented (two at four moves and four at five moves), followed by their yoked control trials. Between each block change there were two practice trials to ensure that the requirements of the current set of tasks had been fully understood.

\section{Pattern recognition}

This test was presented in two phases. Initially, subjects were shown a series of 12 coloured patterns appearing one at a time inside a white box located in the centre of the screen. Each of these 'target' patterns was presented for $3 \mathrm{~s}$, the screen was then cleared and the next pattern appeared. In the second (recognition) phase, 12 pairs of coloured patterns appeared on the screen (one pair at a time) and the subject was required to respond to each pair by touching the pattern they had already seen during the initial (presentation) phase. Each of the target patterns were presented in reverse order and paired with distractor patterns that differed in form but not in colour from the targets. Each response was accompanied by an auditory tone and visual feedback was automatically provided by the computer in the form of green ticks and red crosses. This procedure was then repeated with 12 new patterns and the subject's total score (maximum possible $=$ 24) was expressed as a percentage correct.

\section{Attentional set-shiffing test}

The computerized attentional set-shifting paradigm has also been described in detail elsewhere (Downes et al., 1989; Sahakian et al., 1990; Owen et al., 1991). The subject was required to learn a series of discriminations in which one of two stimulus dimensions was relevant and the other was not, using feedback provided automatically by the computer. Initially, subjects were given a simple simultaneous discrimination in which the stimuli varied along only one of the two 'dimensions' for deriving the stimuli. These dimensions were purple-filled shapes or white lines. The starting dimension was balanced across subjects and groups. A response to one of the two boxes containing the stimuli resulted in an auditory tone, together with visual feedback which informed the subjects of the correctness of their responses. This feedback was in the form of the words 'correct' and 'wrong' presented respectively in green and red lettering above the middle two boxes. The same feedback was used for each of the subsequent stages. After $1.5 \mathrm{~s}$ the screen cleared and an intertrial interval of $1 \mathrm{~s}$ was begun.

Following the initial simple discrimination (SD), the remaining eight stages were as follows. For the second stage (SDR), the discriminations remained the same, but the previously incorrect choice became the correct one and vice versa, i.e. the contingencies were reversed. At the third stage $(C-D)$ the second dimension was introduced with one exemplar of each dimension paired together to form a compound stimulus in two of the response boxes. To succeed, a subject had to continue to respond to the correct exemplar of the previous stage. For this and all subsequent stages, exemplars of the different dimensions were paired in a pseudo-random fashion so that all four possible compound stimuli were used, with the constraint that runs of no more than three trials with the same pairings were allowed. The stimuli for the fourth stage (CD) and subsequent stages were also compounds, but the two exemplars from the different dimensions were superimposed, with the white line always in the foreground. The contingencies were again unchanged from those for the previous two stages. A reversal then occurred at the fifth stage (CDR). New exemplars for both dimensions were introduced at the sixth stage, the intra-dimensional shift (IDS), but the relevant dimension (i.e. shapes or lines) was unchanged from stage 1. This was followed by a further reversal at the seventh stage (IDR). For the penultimate stage, the extra-dimensional shifi (EDS), new exemplars were again introduced, but success at this point depended on the subject shifting response set to the exemplars of the previously irrelevant dimension. Finally, contingencies were reversed to the previously incorrect exemplar of the new dimension (EDR). The main measure of performance on this task was the stage successfully attained. 
Data analysis

The data were analysed using the Statistical Package For The Social Sciences (SPSS/PC) (Nie et al., 1970). One and two-way multivariate analysis of variance (MANOVA) were conducted and were appropriate, Pearson's product moment and Spearman's rank correlation coefficients were calculated.

Preliminary analyses confirmed that there were no significant differences between the three control groups in terms of age, NART IQ or any of the three tests of planning, spatial working memory, spatial span or pattern recognition. The three groups of normal controls were collapsed therefore into a single group of 44 subjects and for each of these tests an orthonormal contrast analysis was performed, comparing each of the medicated Parkinson's disease (mild), medicated Parkinson's disease (severe) and non-medicated Parkinson's disease groups with this single group of normal controls.

In the attentional set-shifting task, the three Parkinson's disease groups were compared with a large group of normal control volunteers $(n=129)$ in terms of the proportion of subjects succeeding or failing to reach criterion at each of the nine stages of the test. The resulting contingency tables were analysed using a likelihood ratio analysis (Kullback, 1959; Robbins, 1977) which allows the use of small cell frequencies, the resulting statistic $2 \mathrm{i}$ being distributed as $\chi^{2}$.

\section{RESULTS}

\section{Spatial short-term memory task}

The Parkinson's disease and control groups were compared in terms of the number of squares that were touched in the correct serial order. Mean values and corresponding standard errors for the span measure are shown in Fig. 2 for the three Parkinson's disease groups and the combined $(n=44)$ groups of controls. An ANOVA showed that the four groups differed significantly on this measure $[\mathrm{F}(3,84)=9.17, P<0.001]$. An orthonormal contrast analysis between the four groups confirmed that the medicated Parkinson's disease (severe) group was significantly impaired on this measure compared with the combined control group $[t(84)=-5.01, P<0.001]$. In contrast, neither the non-medicated Parkinson's disease nor the medicated Parkinson's disease (mild) groups were significantly impaired $[t(84)=-0.01$ and $t(84)=-1.82$, respectively].

\section{Spatial working memory task}

The mean numbers of 'between search errors' made by the three Parkinson's disease groups and the combined control group within each search set size (i.e. four, six or eight boxes) are presented in Fig. 3.

There was a highly significant difference between the four groups $[F(3,84)=9.1$, $P<0.001]$ in terms of the number of between search errors on this task. Orthonormal contrasts between the groups confirmed that both the medicated Parkinson's disease (mild) group $[t(84)=2.77, P<0.01]$ and the medicated Parkinson's disease (severe) group $[t(84)=2.77, P<0.001]$, but not the non-medicated Parkinson's disease group $[t(84)=0.97]$ made significantly more of this type of error than the normal control group. The difference between the two medicated groups of parkinsonian patients was investigated further using the Studentized Newman-Keuls procedure for between group comparisons at each level of task difficulty. The medicated Parkinson's disease (severe) group performed significantly worse than the medicated Parkinson's disease (mild) group only at the most difficult (eight box) level of the test $[\mathrm{F}(1,27)=4.49, P<0.05]$.

When all four groups were compared, there was also a significant main effect of task difficulty $[\mathrm{F}(2,168)=351, P<0.001]$ and a significant interaction between the group and difficulty factors $[\mathrm{F}(6,168)=3.51, P<0.01]$. Contrasts between the groups 


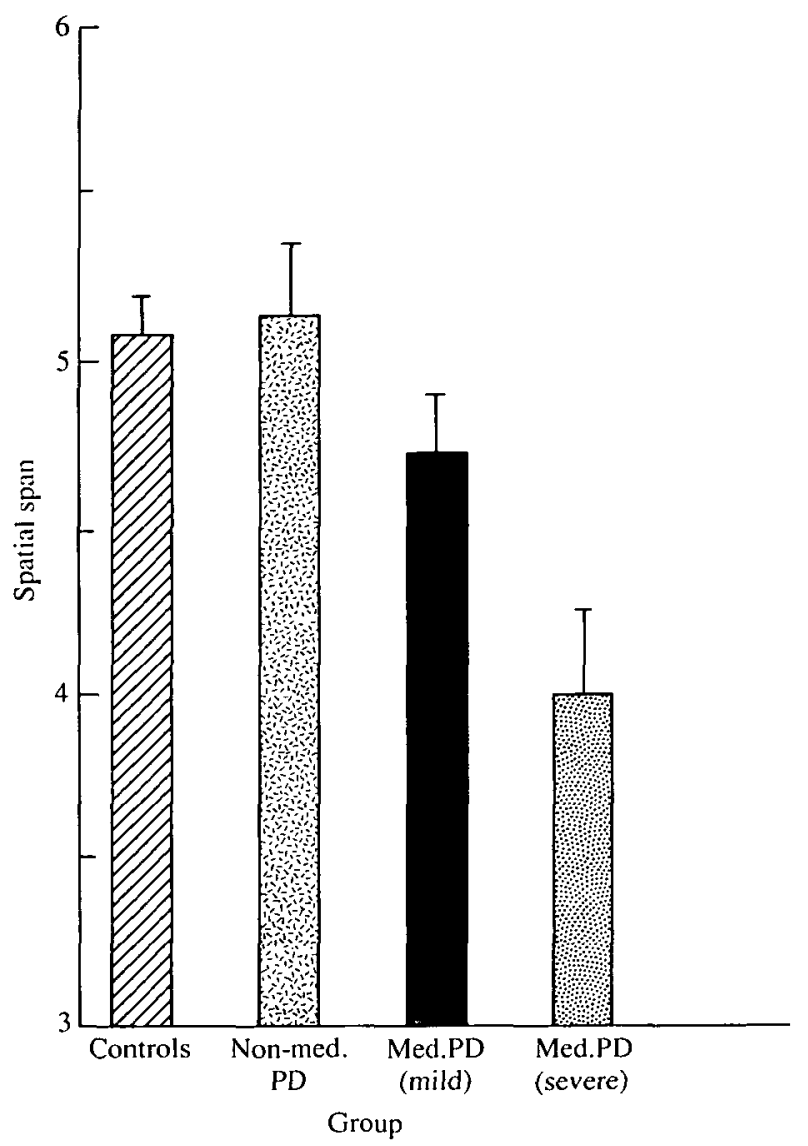

FIG. 2. The mean spatial span for the three patient groups [Non-med. PD = non-medicated Parkinson's disease; Med. PD (mild) = medicated mild Parkinson's disease; Med. PD (severe) = medicated severe Parkinson's disease] and the combined group of control volunteers. Only the medicated group of parkinsonian patients with severe clinical symptoms differ significantly $(P<0.001)$ from controls. Bars represent standard errors of the mean (SEM).

confirmed that this interaction only reached significance when the medicated Parkinson's disease (severe) group was compared with the combined group of normal controls $[t(168)$ $=3.77, P<0.001]$.

The measure of strategy employed in this task was scored on a scale of $1-37$, with lower scores representing more efficient use of the strategy. The best possible score of 1 is obtained when, within each of the more difficult six and eight box problems, the same box is used to initiate each search sequence. Conversely, if every search within each of these problems starts with a different box, the maximum score of 37 is obtained. The mean $( \pm$ SEM) scores for the non-medicated Parkinson's disease, the medicated Parkinson's disease (mild), the medicated Parkinson's disease (severe) groups were 16.6 (1.08), $16.9(1.53)$ and $17.3(0.91)$. The mean score ( \pm SEM) for the combined group of normal controls was $15.75(1.38)$. In all groups there was a significant and similar 


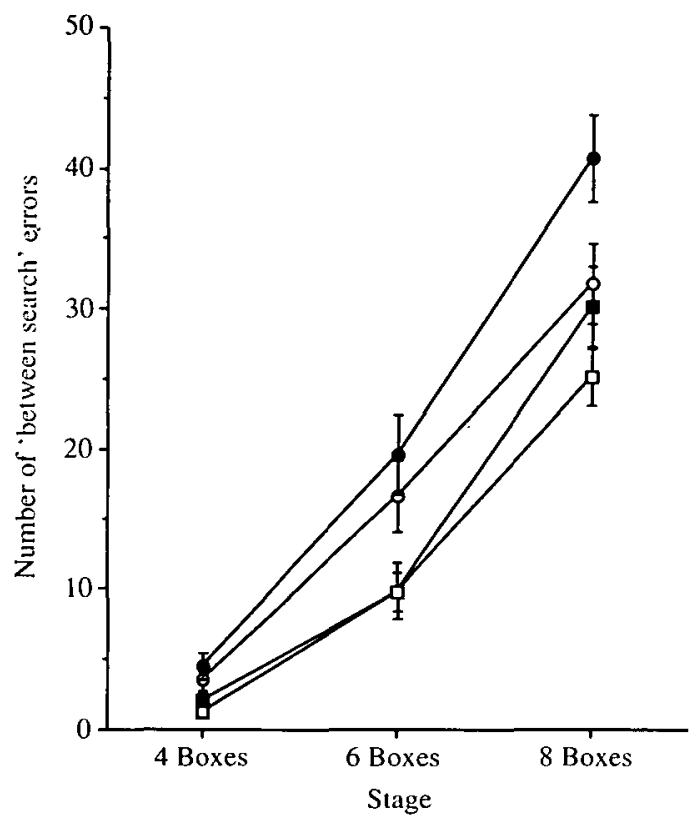

FIG. 3. The incidence of 'between search' errors on the spatial working memory task. Patient groups: $\mathbf{\square}=$ non-medicated Parkinson's disease; $O=$ medicated Parkinson's disease (mild); $\bullet=$ medicated Parkinson's disease (severe); $\square=$ controls. Bars represent SEM $* * P<0.001$.

level of correlation (Pearson's r) between this strategy measure and the number of between-search errors on the more difficult six and eight box problems. However, no significant differences between any of the groups were observed.

These results show that both groups of medicated Parkinson's disease patients made significantly more 'between-search' errors than the control group and at the most difficult level the performance of the medicated Parkinson's disease (severe) group was significantly worse than that of the medicated Parkinson's disease (mild) group. In all patient and control groups, the number of errors in the more difficult problems correlated significantly with the tendency to use a strategy based on a repetitive searching pattern, although the inferior performance of the medicated Parkinson's disease groups could not be attributed to the inappropriate use of this particular search strategy.

\section{Planning task}

Number of moves. Across the 12 test problems, three measures relating to the number of moves required to reach solution were calculated. The 'mean number of moves above the minimum possible' provided a general measure of group performance at each level of difficulty. The 'proportion of problems solved in the minimum number of moves' provided more specific information about task difficulty, and its effect on the four groups. Finally, the 'number of problems solved within the maximum moves allowed', provided a useful index of the subject's ability to solve the problem per se, irrespective of the quality of the performance. 
There was no significant difference between the four groups in terms of the number of moves to solution $[F(3,82)=1.42]$ (see Fig. 4). There was, however, a significant difference between the groups in terms of the number of problems solved in the maximum number of moves allowed $[\mathrm{F}(3,84)=3.29, P<0.05]$, whilst the difference in the proportion of problems solved perfectly approached significance $[\mathrm{F}(3,84)=2.7$, $P=0.051$ ] (see Fig. 4). Comparisons between the groups confirmed that the medicated Parkinson's disease (severe) group was significantly impaired compared with the controls in terms of both these measures of performance accuracy $[t(84)=2.65, P<0.01$ and $t(84)=2.2, P<0.05$, respectively]. In contrast, neither the medicated Parkinson's disease (mild) group nor the non-medicated Parkinson's disease patients were impaired on either of these measures. In all cases, there were significant main effects of task difficulty, although there were no significant interactions between the task difficulty and group factors.

These results confirm that, whilst the non-medicated Parkinson's disease patients and the medicated Parkinson's disease (mild) patients are completely unimpaired in terms of the accuracy of the solutions produced in this planning test, the performance of the

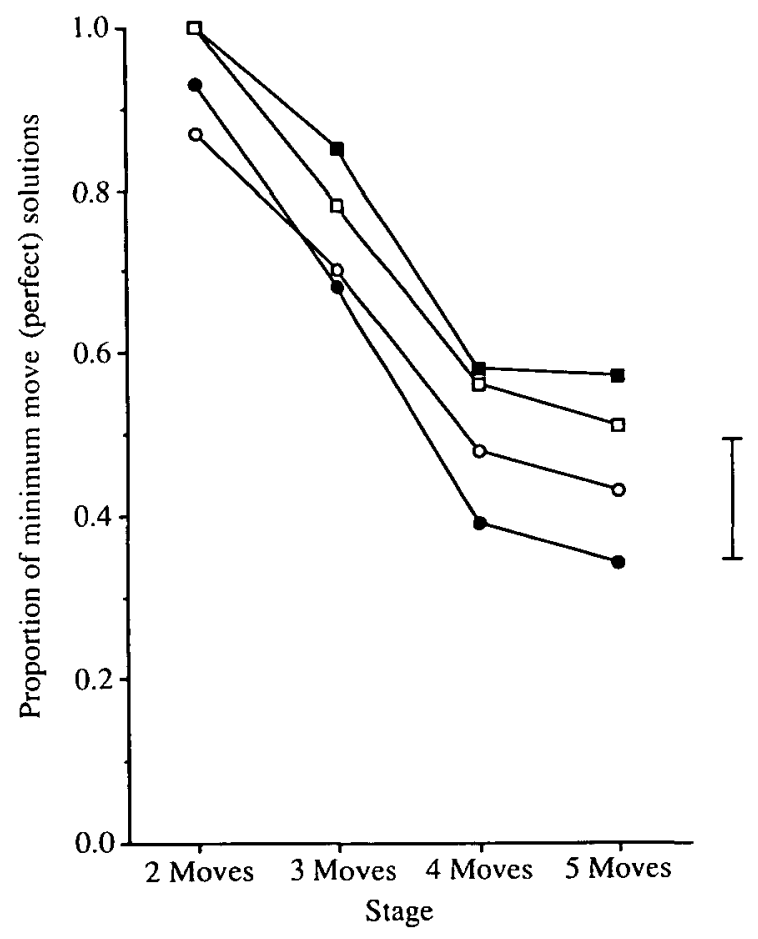

FIG. 4. The Tower of London test (planning accuracy): the proportion of problems solved in the minimum number of moves as a function of difficulty. Patient groups: $\mathbf{Q}=$ non-medicated Parkinson's disease; $\mathrm{O}=$ medicated Parkinson's disease (mild); $\bullet=$ medicated Parkinson's disease (severe); $\square=$ controls. Only the medicated group of Parkinson's disease patients with severe clinical symptoms differ significantly $(P<0.05)$ from controls. Bars represent one standard error of the difference between the means taken from the between group component of the group $\times$ difficulty interaction term of the MANOVA. This is an appropriate index of variation for computing post hoc tests of significance between the mean values of the groups and is calculated according to the formulae provided by Cochran and Cox (1957). 
medicated Parkinson's disease (severe) group is significantly impaired in terms of both the proportion of problems solved in the minimum number of moves (perfect solutions) and the number of problems solved within the maximum number of moves allowed.

Movement times. Baseline measures of motor initiation and motor execution times were extracted from the 12 'yoked control' trials. The motor initiation time represented the mean time between the onset of each problem and the completion of the first selection; that is, a correct touch of the required ball. For the non-medicated Parkinson's disease, medicated Parkinson's disease (mild) and medicated Parkinson's disease (severe) groups of patients, average initial movement times varied between $2.09 \mathrm{~s}$ and $3.49 \mathrm{~s}, 2.78 \mathrm{~s}$ and $3.63 \mathrm{~s}$, and $3.63 \mathrm{~s}$ and $4.73 \mathrm{~s}$, respectively. For the combined control group, the mean initial movement varied between $2.27 \mathrm{~s}$ and $3.46 \mathrm{~s}$. The motor execution time was the time between touching the first ball and completing the sequence of single moves that comprise the whole problem. Since these control problems were 'yoked' to the test problems, the total execution time was divided by the number of moves to give an estimate of the average movement time per move. Mean motor execution times for the three patient groups varied between $2.47 \mathrm{~s}$ and $2.69 \mathrm{~s}, 2.62 \mathrm{~s}$ and $3.26 \mathrm{~s}$, and 3.35 $\mathrm{s}$ and $4.64 \mathrm{~s}$ per move. The corresponding values for the combined control group were $2.11 \mathrm{~s}$ and $2.75 \mathrm{~s}$ per move. When the four groups were compared, there were significant main effects for both initial and subsequent movement time $[\mathrm{F}(3,77)=3.4, P<0.025$ and $\mathrm{F}(3,77)=3.31, P<0.025$, respectively].

A comparison between the medicated Parkinson's disease (severe) group and the combined group of normal controls revealed that both initial movement times $[t(77)$ $=2.96, P<0.01]$ and subsequent movement times $[t(77)=2.97, P<0.01]$ were significantly prolonged in this patient group. In contrast, in the non-medicated Parkinson's disease and the medicated Parkinson's disease (mild) patients neither measure of movement time was significantly different from control values. Comparing all four groups, there was a highly significant main effect of task difficulty [initial movement time: $\mathrm{F}(3,231)=7.22, P<0.001$, subsequent movement time: $\mathrm{F}(3,231)=3.31$, $P<0.025]$ with initial movement time decreasing and subsequent movement time increasing at the more difficult levels. Although the former trend probably represents a practice effect across all groups, the apparent increase in motor execution time with difficulty most likely results from the way in which this estimate was derived (for a more detailed account see Owen et al., 1990). There were no significant interactions between task difficulty and group.

Clearly, across all problems, the medicated Parkinson's disease patients with severe clinical symptoms were impaired at both initiating and executing a simple sequence of moves. This is an important justification for the method of computation used to derive estimates of corrected planning time described below.

Planning time. The movement times discussed above were used to derive estimates of planning or 'thinking time' in the main task. Two separate estimates were calculated. In each problem, the initial thinking time was the time between the presentation of the problem and the first touch, minus the corresponding non-transformed motor initiation time, as calculated from the 'yoked control' task. The subsequent thinking time was the time between the selection of the first ball and the completion of the problem minus the total non-transformed motor execution time derived from the corresponding control problem. Any negative values produced by this subtraction were corrected to zero 
(assuming minimal thinking time), although this was not a common occurrence. Since the subsequent thinking time clearly varied with problem length, this measure was divided by the number of moves to give an estimate of the average thinking time 'per move'. In this way, pure estimates of initial and subsequent 'thinking' or 'planning' time were derived unconfounded by motor initiation or execution times. The mean initial thinking times for the three groups of patients with Parkinson's disease and the combined control group are shown in Fig. 5.

There was a highly significant difference between the four groups on this measure of 'thinking' or planning time $[\mathrm{F}(3,82)=4.48, P<0.01]$. Both the medicated Parkinson's disease (mild) and the medicated Parkinson's disease (severe) groups of patients had significantly prolonged initial thinking times when compared with the combined group of normal controls $[t(82)=2.35, P<0.025$ and $t(82)=2.18$, $P<0.05$, respectively]. However, the contrast between the non-medicated Parkinson's disease group and the control group did not reach significance $[t(82)=-1.23$, $P=0.22$ ]. There was a significant main effect of task difficulty across the four groups $[\mathrm{F}(3,246)=38.17, P<0.001]$ and no significant interaction between the group and task difficulty factors.

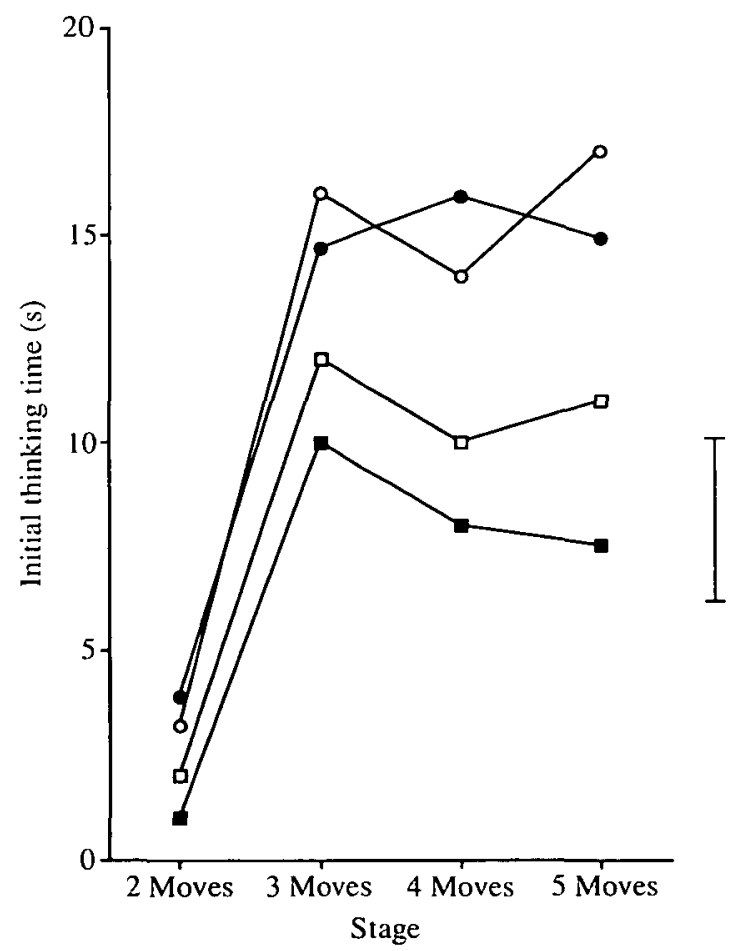

FIG. 5. The Tower of London test (planning time): the average initial thinking (planning) times prior to the first move as a function of difficulty. Patient groups: $\mathbf{Q}=$ non-medicated Parkinson's disease; $\mathrm{O}=$ medicated Parkinson's disease (mild); $\bullet=$ medicated Parkinson's disease (severe); $\square=$ controls. Bars represent one standard error of the difference between the means (see legend to Fig. 4). 
These results confirm that whilst initial thinking or 'planning' time is unaffected in the mildly affected, non-medicated patients with Parkinson's disease, this measure is significantly prolonged in both groups of medicated patients who are later in the course of the disease.

When the subsequent thinking times at each level of difficulty were analysed there was no significant difference between the patient groups and the combined group of normal controls $[\mathrm{F}(3,77)=0.41]$. There was a significant effect of task difficulty $[\mathrm{F}(3,231)=18.14, P<0.001]$ and no significant interaction between the difficulty and the group factors.

Thus, in contrast to the observed differences in initial thinking time, there were no differences between the three Parkinson's disease groups and the combined control group in the amount of time spent thinking about the planning problems subsequent to making the first move.

\section{Pattern recognition}

Mean values (percentage correct) and corresponding standard errors in parentheses for the pattern recognition task were: controls $86.6 \%$ (1.86), non-medicated Parkinson's disease $85.8 \%$ (3.48), medicated Parkinson's disease (mild) $84.1 \%$ (3.86), medicated Parkinson's disease (severe) $80.0 \%$ (2.66). An ANOVA showed that the four groups did not differ significantly on this test of pattern recognition $[\mathrm{F}(3,62)=1.29, P=0.28]$.

In contrast to their marked differences on the tests of planning, spatial working memory, and short-term spatial span the three groups of patients with Parkinson's disease did not differ in terms of their short-term recognition memory for patterns.

\section{Attentional set-shifting test}

Figure 6 shows the cumulative percentage of subjects succeeding at each stage of the attentional set-shifting test. As can be seen, $77 \%$ of control subjects successfully completed all stages of the test compared with $46 \%$ of the non-medicated Parkinson's disease group, $55 \%$ of the medicated Parkinson's disease (mild) and only $11 \%$ of the medicated Parkinson's disease (severe) groups. Likelihood ratio analysis of the contingency tables for success and failure confirmed that there were significant differences among the four groups $(2 \mathrm{i}=20.98$, d.f. $=3, P<0.001)$. Further orthogonal contrasts showed that the controls were significantly superior to the combined Parkinson's disease groups $(2 \mathrm{i}=18.78$, d.f. $=1, P<0.001)$, although the three Parkinson's disease groups could not be differentiated. Additional analysis was made in order to identify the stages at which the differences in cumulative failure became significant. Attention was focused on the transition between the stages at which the subject had to form and maintain response set (i.e. up to and including the IDS and IDR) and the specific stage when attentional set-shifting was required (i.e. at the EDS). In cumulative terms there was a significant difference among the four groups by the IDR stage $(2 \mathrm{i}=12.54$, d.f. $=3, P<0.01)$ and again this was attributable to a significant difference between the controls and the three Parkinson's disease groups $(2 \mathrm{i}=10.61$, d.f. $=1, P<0.01)$. A final set of comparisons was made specifically at the EDS stage, including only those subjects able to attempt that stage. These showed once again that the four groups differed significantly $(2 \mathrm{i}=14.06$, d.f. $=3, P<0.01)$ and that the three Parkinson's disease groups were significantly worse than the controls $(2 \mathrm{i}=9.79$, d.f. $=1, P<0.01)$. A final orthogonal 


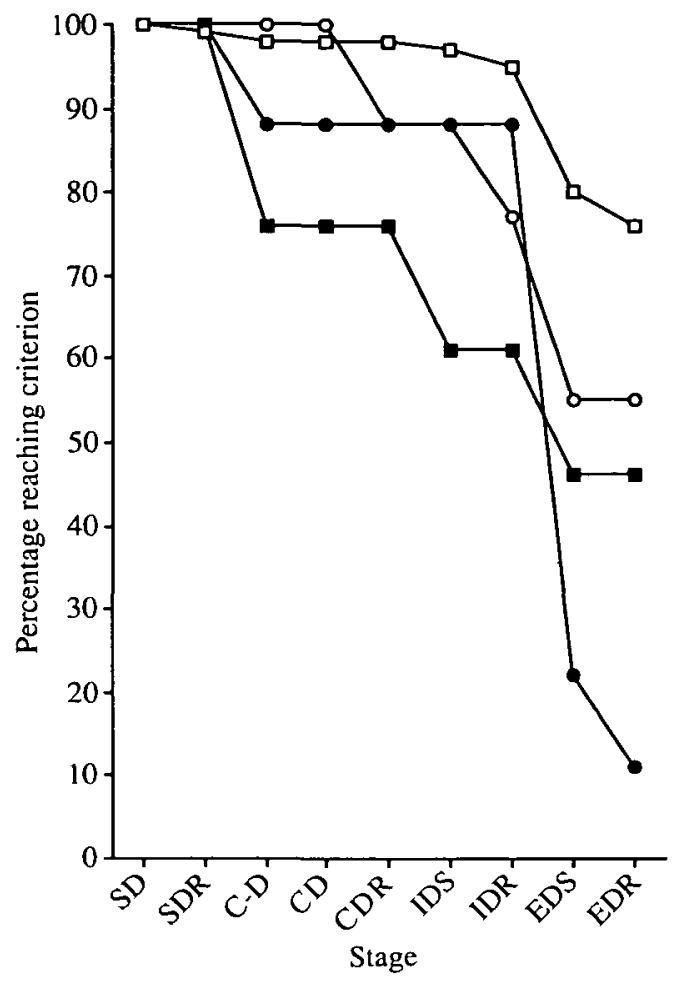

FIG. 6. The percentage of subjects in each group [patients: $\square=$ non-medicated Parkinson's disease; $O=$ medicated Parkinson's disease (mild); $\bullet=$ medicated Parkinson's disease (severe); $\square=$ controls] reaching criterion at each of the nine stages of the discrimination learning paradigm. (For stages see text under Attentional set-shiffing test in Methods.)

contrast confirmed for this stage that the impairment in the medicated Parkinson's disease (severe) group was greater than in the other two Parkinson's disease groups combined $(2 \mathrm{i}=4.23$, d.f. $=1, P<0.05)$.

In summary, all three Parkinson's disease groups were severely impaired at the attentional set-shifting task, although this deficit was not specifically limited to the ability to shift attention between competing stimulus dimensions, since cumulative impairments were evident even prior to this stage of the test.

\section{Correlational analysis}

Depression score. In order to assess the relationship between depression and performance on the computerized tests, Pearson's product moment correlation coefficients were calculated between the GDS score and the main performance indices described above. In the two groups of medicated patients, the GDS depression measure correlated highly with initial movement time at levels 3 and $5(r=0.58, P<0.01 ; r=0.56$, $P<0.01)$ and with subsequent movement time at levels $2,3,4$ and $5(\mathrm{r}=0.61$, $P<0.01 ; \mathrm{r}=0.56, P<0.01 ; \mathrm{r}=0.68, P<0.01 ; \mathrm{r}=0.67, P<0.01$ ). In contrast, there were no significant correlations between the depression measure and estimates of initial or subsequent thinking time. 
Interrelationships among cognitive tests. To assess the general relationships among the five tests described here Spearman's rank correlation $\left(r_{s}\right)$ coefficients (including partial correlations) were calculated between several indices of performance, derived from the main variables described above. This non-parametric correlation coefficient was used because of the ordinal nature of the 'stage successfully completed' variable in the attentional set-shifting test. Specifically, span, total 'between search errors' (spatial working memory test), number of problems solved perfectly (Tower of London), mean initial and subsequent thinking time (Tower of London), mean initial and subsequent movement time (Tower of London), total pattern recognition score and last stage successfully completed in the attentional set-shifting paradigm were intercorrelated. Separate intercorrelation matrices were generated for those subjects from the group of combined parkinsonian patients and the matched group of 44 controls, who completed all of the tests.

Among control subjects, accuracy on the Tower of London test (number of perfect solutions) was positively related to initial thinking time $\left(\mathrm{r}_{s}=0.41, P<0.05\right)$ and negatively related to subsequent thinking time $\left(r_{s}=-0.41, P<0.05\right)$. These correlations were robust against partial correlations with other factors. In contrast, no significant relationships existed among these variables in the combined Parkinson's disease group $\left(\mathrm{r}_{s}=0.09\right.$ and $\mathrm{r}_{s}=-0.01$, respectively).

In both controls and parkinsonian patients there was a significant correlation between the total errors score on the spatial working memory test and the measure of accuracy (perfect solutions) on the Tower of London test $(\mathrm{r}=-0.64, P<0.01$ and $\mathrm{r}=-0.67$, $P<0.01$, respectively). Again, these correlations were robust against partial correlations with other factors. By contrast, the measures of pattern recognition and attentional setshifting ability did not correlate significantly with any of the other factors, in either the control or the Parkinson's disease groups.

\section{DISCUSSION}

The results of this investigation have considerable significance for the staging of cognitive decline in Parkinson's disease. This study has demonstrated that groups of patients at different stages of Parkinson's disease can be differentiated in terms of their performance on tests of spatial working memory, spatial short-term memory and high level planning ability but not in terms of short-term pattern recognition memory. In a computerized version of the Tower of London test of planning, an impairment in terms of the accuracy of the solution produced was only evident in a group of medicated Parkinson's disease patients with severe clinical symptoms. These patients completed significantly fewer problems in the minimum number of moves (perfect solutions) and solved fewer problems within the maximum number of moves allowed. Although none of the three Parkinson's disease groups was impaired, in terms of the amount of time spent thinking subsequent to the first move ('subsequent thinking time'), the medicated patients with both mild and severe clinical symptoms had significantly prolonged initial thinking (planning) times prior to making the first move.

The results of this study both confirm and extend the preliminary findings of Morris et al. (1988) in which prolonged initial thinking (planning) times were demonstrated in a group of 12 medicated patients with Parkinson's disease using an earlier version 
of the computerized Tower of London test. This performance is most consistent with the group of medicated Parkinson's disease patients with mild clinical symptoms described in the present study, who also showed prolonged initial thinking times on the more recent form of this test. It is important to note that the mean Hoehn and Yahr score in this group (1.8) is rather similar to the mean score from the 12 patients included in the earlier study (2.17) (Morris et al., 1988).

In the present study, the observations of Morris et al. (1988) have been extended in three important ways. First, we have extended the analysis to include other stages of Parkinson's disease. Secondly, by using a version of the test which has already been employed to identify a specific pattern of deficits in neurosurgical patients with frontal lobe excisions, we have been able to make direct comparisons between the two conditions. Thirdly, we have included a measure of depression which has previously been shown to contribute to the cognitive profile of parkinsonian patients (Starkstein et al., 1989).

Our group of medicated patients in the more advanced stages of Parkinson's disease were impaired in terms of the accuracy of the solution produced as well as showing prolonged initial thinking times. Importantly, this group had a greater mean Hoehn and Yahr clinical disability score (3.43) than the patients described by Morris et al. (1988). The present investigation also included a group of parkinsonian patients who were nonmedicated and who, for the most part, had mild clinical symptoms (mean score 1.93). This patient group were completely unimpaired on this test of planning.

The Tower of London test has previously been shown to be sensitive to frontal lobe dysfunction in a group of neurosurgical patients with localized excisions of the frontal lobes (Owen et al., 1990). The frontal lobe patients required significantly more moves to solve the problems and, like the parkinsonian patients with more severe clinical symptoms, produced fewer perfect solutions (Owen et al., 1990). In addition, the motor control condition revealed that movement times were significantly increased in the frontal lobe group, which again is similar to the pattern observed in the medicated Parkinson's disease (severe) group in the present study. However, unlike both groups of medicated Parkinson's disease patients, the frontal lobe group was unimpaired in the initial thinking (planning) time but did spend a significantly longer time thinking subsequent to the first move. Therefore, although some patients with Parkinson's disease (dependent upon the severity of symptoms) are impaired in this test of planning, the qualitative nature of this deficit appears to be different from that seen in patients with frontal lobe damage. In particular, initial thinking time was significantly prolonged in both groups of medicated Parkinson's disease patients, but unaffected in patients with frontal lobe damage. Conversely, subsequent thinking time was prolonged in the frontal lobe patients, but unaffected in patients with Parkinson's disease. The diminished accuracy of planning found in the frontal lobe patients (perfect solutions) was only evident in the most severely affected group of parkinsonian patients.

Prolonged initial thinking time in patients with Parkinson's disease, a possible correlate of 'bradyphrenia', may reflect a state of psychomotor retardation characteristic of Parkinson's disease and depressed patients. Rogers et al. (1987) proposed that bradyphrenia in Parkinson's disease and psychomotor retardation in primary depressive illness may be closely related and that the dopaminergic system may be involved in both. In the present study, the depression score was not correlated with any aspect of planning performance; in fact, it only correlated significantly with speed of performance in the 
motor control condition, thus confirming recent conclusions of Cooper et al. (1991). The former observation suggests that depression in Parkinson's disease does not contribute to the pattern of deficits in planning that we have observed. This conclusion is supported by the recent finding that elderly depressed patients show no evidence of slowed initial or subsequent thinking time in exactly the same test (B. Beats et al., unpublished results).

The prolonged initial thinking time in the medicated patients with Parkinson's disease can be interpreted in a number of ways. First, the slow, but generally accurate, performance of the patients with mild clinical disability may reflect a speed-error tradeoff. For example, in control subjects significant correlations exist between performance accuracy (perfect solutions) and measures of initial and subsequent thinking time. Specifically, prolonged initial thinking time is related to improved performance, but prolonged subsequent thinking time to inferior performance. Hence, optimal performance can be attained by thinking before one initiates a solution rather than during its execution. However, in the study of Morris et al. (1988) and in the present investigation, analysis revealed no obvious relationship between the accuracy and latency of thinking in parkinsonian patients. Furthermore, although the two medicated Parkinson's disease groups were equally slow in initiating their solutions, they differed significantly in their accuracy of performance. Prolonged initial thinking times may also be attributed to mental activity that is not devoted to solving the problem at hand, resulting in 'blanking' or 'mind-wandering' (Watts et al., 1988). The former may be likened to the so-called 'psychic akinesia' occasionally reported in cases of Parkinson's disease (Hassler, 1953; Rogers, 1986). Finally, slowed thinking time may reflect delays in switching between different representations of the goal and of the schemata governing the sequences of moves under consideration (Morris et al., 1990; see also the hypothesis discussed by Sagar et al., 1988b, p. 535). This latter hypothesis is relevant to theories of cognitive dysfunction in Parkinson's disease which focus on deficits in attentional set-shifting ability, possibly resulting from fronto-striatal dysfunction (e.g. Cools et al., 1984). A recent study (Downes et al., 1989) has shown that the impairment in extra-dimensional set-shifting performance in patients with Parkinson's disease is at least as great in unmedicated, early in the course patients as in medicated patients with relatively mild clinical symptoms. In the present study, these observations were confirmed and extended in a different population of parkinsonian patients. Whilst deficits were observed in all three Parkinson's disease groups, the medicated Parkinson's disease (severe) group were significantly more impaired at the EDS stage than the medicated Parkinson's disease (mild) and non-medicated Parkinson's disease groups. This profile of progressively greater cognitive impairment with increased severity of clinical symptoms is thus similar to that seen in the tests of planning and spatial working memory. However, of the three 'frontal lobe' tasks only this test of attentional set-shifting ability revealed significant deficits in the non-medicated Parkinson's disease group. It should be noted that the parkinsonian patients were not only impaired at shifting set in this paradigm, but also at those earlier stages of the test requiring the formation and maintenance of a response set for the relevant stimulus dimension. Similarly, Cooper et al. (1991) have recently demonstrated selective deficits in set formation in early, untreated patients with Parkinson's disease using the Wisconsin Card Sorting Test. Moreover, this apparent impairment of set stability is not merely a function of test sensitivity or global cognitive impairment because patients early in the course of Alzheimer's disease successfully 
completed the present set-shifting task despite showing deficits in visual memory performance (Sahakian et al., 1990).

Spatial working memory and spatial span may also play roles in effective planning on the Tower of London test. In the present study, both groups of medicated Parkinson's disease patients were significantly impaired in a test of spatial working memory recently shown to be sensitive to frontal lobe damage (Owen et al., 1990), whilst the unmedicated Parkinson's disease group performed at the same level as controls. Some of the deficits in planning function observed in these patients may be attributable to impaired working memory processes which may be vital in the initial search for possible solutions. The spatial working memory impairment in mildly affected medicated Parkinson's disease patients in this study contrasts with the results of Morris et al. (1988) who found no impairment, using an earlier version of this test. Although the precise reasons for this discrepancy are unclear, the current version of the test is designed to discourage the acquisition of strategies based on repetitive search sequences which may previously have compensated for impaired performance in the Parkinson's disease group. In the present study no significant strategy deficits were apparent in any of the Parkinson's disease groups, although, using the same task, strategies have been shown to be impaired in patients with frontal lobe damage (Owen et al., 1990).

Since the Tower of London test requires sequences of up to five moves, it is possible that planning deficits could arise from an impairment in the ability to reproduce a stored sequence of spatial moves. In the test of short-term spatial span, designed to assess this ability, no deficits were found in the two Parkinson's disease groups with relatively mild clinical disability. However, deficits were apparent in the severely affected Parkinson's disease group and this could have affected the accuracy of their solutions in the Tower of London test of planning. Reduced spatial span is not characteristic of patients with frontal damage (Milner, 1971; Owen et al., 1990), but is observed following right-sided temporal lobe lesions that include the hippocampus (Milner, 1971), and in Alzheimer's disease (Corkin et al., 1984; Sullivan et al., 1986). Thus, impaired spatial span in the medicated Parkinson's disease patients with more severe clinical symptoms may represent a more global deterioration of cognition in this group.

The results of this cross-sectional study have considerable significance for the possible progression of cognitive decline in patients with Parkinson's disease. As Table 2 shows, there is an apparent increase in severity and broadening of cognitive impairments as patients show increasing clinical disability (reflected in their Hoehn and Yahr ratings) which may merit further investigation in a longitudinal design. Because of the controlled nature and design of these tests, these cognitive deficits cannot simply be explained in terms of motor dysfunction. Moreover, it seems likely that some cognitive deficits, particularly those resembling frontal lobe dysfunction, progress in parallel with the motor deficits characteristic of Parkinson's disease, and may reflect differing forms of neuropathological involvement. This apparent 'progression' on tests sensitive to frontal lobe damage could simply reflect a global difference in cognitive capacity between the patients with mild and severe Parkinson's disease. This is unlikely, however, since the three Parkinson's disease groups could not be distinguished in terms of their performance on the test of pattern recognition memory. This test is not sensitive to frontal lobe excisions (A. M. Owen et al., unpublished results), although significant deficits have been observed in other Parkinson's disease groups and patients with dementia of Alzheimer type with 
TABLE 2. SUMMARY OF RESULTS

\begin{tabular}{|c|c|c|c|c|}
\hline & $\begin{array}{c}\text { Non-medicated } \\
\text { Parkinson's disease }\end{array}$ & $\begin{array}{c}\text { Medicated } \\
\text { Parkinson's disease } \\
\text { (mild) }\end{array}$ & $\begin{array}{c}\text { Medicated } \\
\text { Parkinson's disease } \\
\text { (severe) }\end{array}$ & $\begin{array}{l}\text { Frontal } \\
\text { lobe }\end{array}$ \\
\hline Pattern recognition & $\sqrt{ }$ & $\sqrt{ }$ & $\sqrt{ }$ & $\checkmark$ \\
\hline Span & $\checkmark$ & $\sqrt{ }$ & $X$ & $\sqrt{ }^{*}$ \\
\hline $\begin{array}{l}\text { Spatial working memory } \\
\text { ('between search' errors) }\end{array}$ & $\sqrt{ }$ & $\mathrm{X}$ & $\mathrm{X}$ & $\mathrm{X}^{*}$ \\
\hline $\begin{array}{l}\text { Minimum move solutions } \\
\text { (Tower of London) }\end{array}$ & $\sqrt{ }$ & $\sqrt{ }$ & $x$ & $\mathrm{X}^{*}$ \\
\hline $\begin{array}{l}\text { Initial thinking time } \\
\text { (Tower of London) }\end{array}$ & $\sqrt{ }$ & $x$ & $x$ & $\sqrt{ }^{*}$ \\
\hline $\begin{array}{l}\text { Subsequent thinking time } \\
\text { (Tower of London) }\end{array}$ & $\checkmark$ & $\checkmark$ & $\checkmark$ & $X^{*}$ \\
\hline Attentional set-shifting & $\mathrm{x}$ & $\mathrm{x}$ & $\mathrm{x}$ & $\mathrm{X}^{* *}$ \\
\hline
\end{tabular}

mild and moderate clinical symptoms (Sahakian et al., 1990; Saghal et al., 1991). In the former study, several of the medicated Parkinson's disease patients had relatively poor scores on the KOLT (Kendrick, 1985) (although they were not formally diagnosed as clinically demented) and this was associated with their poor recognition memory performance. By comparison, in the present study, any parkinsonian patient scoring below the accepted cut-off for dementia ( $<23$ on the KOLT or $<24$ on the MMSE) was not tested.

One factor that complicates our understanding of the course of cognitive decline in Parkinson's disease is the potential role of medication. It is possible that some of the deficits observed may be attributable to various aspects of medication, as both L-dopa (Gotham et al., 1988) and scopolamine (Dubois et al., 1987) have been shown to affect cognitive performance in Parkinson's disease patients. However, medication clearly cannot account for the deficits in the visual discrimination learning paradigm seen in the non-medicated Parkinson's disease group in this study and in the previous study by Downes et al. (1989). It is also unlikely that dopaminergic medication disrupts performance in the two medicated Parkinson's disease groups included in this investigation since a recent study (Lange et al., 1992) has demonstrated that L-dopa withdrawal produces large deficits in each of the 'frontal lobe' tests used here, as well as impairing spatial span but leaving pattern recognition memory unaffected.

The contribution of fronto-striatal mechanisms to cognitive deficits in Parkinson's disease

Whilst each of the tests of planning, attentional set-shifting and spatial working memory appear to be sensitive to damage in the frontal cortex, they may depend upon differential involvement within this structure. Experimental lesions in non-human primates performing analogous versions of some of these tasks suggest that spatial working memory is especially sensitive to dorsolateral frontal damage (Brozoski et al., 1979), whereas set-shifting may be dependent on orbitofrontal (Passingham, 1972) as well as dorsolateral (Milner, 1964) regions. Moreover, in view of the intimate circuitry linking the striatum to this area, via parallel, segregated cortico-striatal loops that feed back via the pallidum 
and thalamus to the frontal cortex (Alexander et al., 1986), task performance may be compromised by striatal pathology disrupting the functions of these neural loops. In particular, it is known that damage to different regions of the caudate nucleus produces cognitive deficits which resemble the effects of damage to their corresponding targets of projection within the prefrontal cortex (e.g. Divac et al., 1967). As Parkinson's disease is associated with striatal dopamine depletion that affects both the caudate nucleus, as well as the putamen, and also with dopamine depletion in the prefrontal cortex (Scatton et al., 1983), it is possible that the 'frontal like' deficits reported here result from either or both of these forms of pathology. Support for this is provided by the recent findings of selective exacerbation of the deficits in the present 'frontal' tests by L-dopa withdrawal in Parkinson's disease (Lange et al., 1992). In this latter study, visual memory and learning (including the pattern recognition test used here) were not impaired by L-dopa withdrawal. Thus, it is possible that the deficits in visual memory and learning that can accompany severe Parkinson's disease might be related to non-dopaminergic pathology, for example in the cortical deafferentation of serotonergic, noradrenergic and cholinergic neurotransmitter systems (Agid et al., 1987).

Taken together, the results of this study suggest that non-medicated patients with mild clinical symptoms are impaired on only one of three tests shown to be sensitive to frontal lobe damage (see Table 2). Although they show deficits in attentional set-shifting ability, they are unimpaired on the tests of spatial working memory and planning. This may suggest a limited anatomical focus for the cognitive impairments occurring early in the course of Parkinson's disease. A similar hypothesis has been proposed by Freedman and Oscar-Berman (1986) to account for different patterns of deficits on tasks differentially sensitive to lesions in different parts of the frontal cortex. In contrast, medicated patients with Parkinson's disease were impaired on all three tests sensitive to frontal lobe function, although the nature of the deficits were different in both the Tower of London test of planning and the test of spatial working memory. One obvious implication of this apparent progression of deficits is that more extensive regions of fronto-striatal circuitry become disrupted later in the course of the disease.

The precise neural mediation of cognitive functions as complex as planning are not known, although it seems likely that it involves the integration of several different component processes, including working memory, set formation, maintenance and shifting and other executive functions (e.g. goal evaluation and sequencing). This in turn would involve the co-ordination of different streams of information processing in the postulated parallel cortico-striatal loops. Preliminary evidence bearing on the unitary (or otherwise) nature of the cognitive requirements of the main tests used here can be gleaned from the correlational analysis of the interrelationships among the various tests. These analyses suggested that there is a significant association between the spatial working memory task and the accuracy of planning on the Tower of London test, confirming that working memory is a major contributor to some aspects of planning on this task. By contrast, the measures of pattern recognition and attentional set-shifting failed to intercorrelate with any of the other variables, suggesting that these tests assess functions that are somewhat independent of planning ability. Therefore, in Parkinson's disease the fact that deficits in attentional set-shifting appear to occur before those in planning does not have any causal implications.

The fact that planning function in Parkinson's disease is impaired in a different manner 
from that in frontal lobe patients suggests that the study of these different disorders may enable us to dissociate different aspects of task performance which may depend upon independent elements within functional neural loops. For example, accuracy and efficiency of planning may be mediated largely by mechanisms within the frontal lobes themselves, whilst speed of thinking may be modulated by neurotransmitter systems of subcortical origin that innervate either or both the prefrontal cortex or the caudate nucleus. In normal subjects these facets of performance are in balance, producing optimal trade-off between speed and accuracy. However, this balance appears to be disrupted in patients with Parkinson's disease or frontal lobe damage, though in rather different ways, possibly reflecting altered cortico-striatal interactions consequent upon striatal dopamine depletion or the partial loss of cortical input to the striatum, respectively. This and similar informative comparisons between groups of patients with different pathology of the cortico-striatal circuitry are thus enabling us to identify the precise role of its various elements in complex cognitive functions.

\section{ACKNOWLEDGEMENTS}

We wish to thank Dr J. L. Evenden for preparing the original programs and Professor P. Rabbitt for allowing us access to control subjects drawn from the North East Age Research panel at Newcastle University. This research was supported by a Programme Grant from the Wellcome Trust to T. W. Robbins, B. J. Everitt and B. J. Sahakian.

\section{REFERENCES}

Agid Y, Ruberg M, Dubois B, Pillon B (1987) Anatomoclinical and biochemical concepts of subcortical dementia. In: Cognitive Neurochemistry. Edited by S. M. Stahl, S. D. Iversen and E. C. Goodman. Oxford: Oxford University Press, pp. 248-271.

Alexander GE, DeLong MR, Strick PL (1986) Parallel organization of functionally segregated circuits linking basal ganglia and cortex. Annual Review of Neuroscience, 9, 357-381.

Boller F, Passafiume D, Keefe NC, Rogers K, Morrow L, Kim Y (1984) Visuospatial impairments in Parkinson's disease: role of perceptual and motor factors. Archives of Neurology, Chicago, 41, $485-490$.

Bowen FP, Kamienny RS, Burns MM, Yahr MD (1975) Parkinsonism: effects of levodopa treatment on concept formation. Neurology, Minneapolis, 25, 701-704.

BRown RG, MARSDEN CD (1988) 'Subcortical dementia': the neuropsychological evidence. Neuroscience, 25, 363-387.

Brown RG, MARSDEN CD, QUINN N, WYKE MA (1984) Alterations in cognitive performance and affectarousal state during fluctuations in motor function in Parkinson's disease. Journal of Neurology, Neurosurgery, and Psychiatry, 47, 454-465.

Brozoski TJ, Brown RM, Rosvold HE, Goldman PS (1979) Cognitive deficit caused by regional depletion of dopamine in prefrontal cortex of rhesus monkey. Science, 205, 929-932.

Canavan aGM, Passingham Re, Marsden CD, Quinn N, Wyke M, Polkey CE (1989) The performance on learning tasks of patients in the early stages of Parkinson's disease. Neuropsychologia, $27,141-156$.

Cochran WG, Cox GM (1957) Experimental Designs. Second edition. New York: John Wiley.

Cools AR, van den Bercken JHL, Horstink MWI, van Spaendonck KPM, Berger HJC (1984) Cognitive and motor shifting aptitude disorder in Parkinson's disease. Journal of Neurology, Neurosurgery, and Psychiatry, 47, 443-453.

Cooper Ja, Sagar JH, Jordan N, Harvey NS, Sullivan EV (1991) Cognitive impairment in early, untreated Parkinson's disease and its relationship to motor disability. Brain, 114, 2095-2122. 
Corkin S, Growdon JH, Nissen MJ, Huff FJ, Freed DM, Sagar HJ (1984) Recent advances in the neuropsychological study of Alzheimer's disease. In: Alzheimer's Disease: Advances in Basic Research and Therapies. Edited by R. J. Wurtman, S. H. Corkin and J. H. Growdon. Cambridge, MA: Center for Brain Sciences and Metabolism Charitable Trust, pp. 75-93.

Divac I, Rosvold HE, Szwarcbart MK (1967) Behavioral effects of selective ablation of the caudate nucleus. Journal of Comparative and Physiological Psychology, 63, 184-190.

Downes JJ, Roberts AC, Sahakian BJ, Evenden JL, Morris RG, Robiins TW (1989) Impaired extradimensional shift performance in medicated and unmedicated Parkinson's disease: evidence for a specific attentional dysfunction. Neuropsychologia, 27, 1329-1343.

Dubois B, Danzé F, Pillon B, Cusimano G, Lhermitre F, Agid Y (1987) Cholinergic-dependent cognitive deficits in Parkinson's disease. Annals of Neurology, 22, 26-30.

Flowers KA, RoberTson C (1985) The effect of Parkinson's disease on the ability to maintain a mental set. Journal of Neurology, Neurosurgery, and Psychiatry, 48, 517-529.

Folstein MF, Folstein SE, MCHugh PR (1975) 'Mini-Mental State': a practical method for grading the cognitive state of patients for the clinician. Journal of Psychiatric Research, 12, 189-198.

Freedman M, Oscar-Berman M (1986) Selective delayed response deficits in Parkinson's and Alzheimer's disease. Archives of Neurology, Chicago, 43, 886-890.

Gibson JJ (1941) A critical review of the concept of set in contemporary experimental psychology. Psychological Bulletin, 38, 781-817.

Gotham AM, Brown RG, MARSDEN CD (1988) 'Frontal' cognitive function in patients with Parkinson's disease 'on' and 'off' levodopa. Brain, 111, 299-321.

HASSLER R (1953) Extrapyramidal-motorische Systeme and Erkrankungen. In: Handbuch der inneren Medezin, Volume 3. Edited by G. von Bergmann and R. Staehelin. Berlin: Springer, pp. 676-904.

HoEHN MM, YAHR MD (1967) Parkinsonism: onset, progression, and mortality. Neurology, Minneapolis, $17,427-442$.

KendRICK DC (1985) Kendrick Cognitive Tests For the Elderly. Windsor: NFER-Nelson.

Kullback S (1959) Information Theory and Statistics. New York: John Wiley.

Lange KW, Robbins TW, Marsden CD, James M, Owen AM, Paul GM (1992) L-Dopa withdrawal in Parkinson's disease selectively impairs cognitive performance in tests sensitive to frontal lobe dysfunction. Psychopharmacology, 107, 394-404.

LeEs AJ, SMITH E (1983) Cognitive deficits in the early stages of Parkinson's disease. Brain, 106, 257-270.

MiLner B (1964) Some effects of frontal lobectomy in man. In: The Frontal Granular Cortex and Behavior. Edited by J. M. Warren and K. Akert. New York: McGraw-Hill, pp. 313-331.

MiLNER B (1971) Interhemispheric differences in the localization of psychological processes in man. British Medical Bulletin, 27, 272-277.

Morris RG, Downes JJ, Sahakian BJ, Evenden Jl, Heald A, Robiins TW (1988) Planning and spatial working memory in Parkinson's disease. Journal of Neurology, Neurosurgery, and Psychiatry, 51, $757-766$.

MorRis RG, Downes JJ, Robins TW (1990) The nature of the dysexecutive syndrome in Parkinson's disease. In: Lines of Thinking. Reflections on the Psychology of Thought, Volume 2. Edited by K. J Gilhooly, M. T. G. Keane, R. H. Logie and G. Erdos. Chichester: John Wiley, pp. 247-258.

Mortimer JA, Pirozzolo FJ, Hansch EC, Webster DD (1982) Relationship of motor symptoms to intellectual deficits in Parkinson's disease. Neurology, New York, 32, 133-137.

NELSON HE (1982) National Adult Reading Test (NART): Test Manual. Windsor: NFER-Nelson.

Nie NH, Hadlai Hull C, Jenkins JG, Steinbrenner K, Bent DH (1970) Statistical Package for the Social Sciences. New York: McGraw-Hill.

Ogden JA, Growdon JH, CoRkin S (1990) Deficits on visuospatial tests involving forward planning in high-functioning parkinsonians. Neuropsychiatry, Neuropsychology and Behavioral Neurology, 3, $125-139$.

Owen AM, Downes JD, Sahakian BJ, Polkey CE, Robbins TW (1990) Planning and spatial working memory following frontal lobe lesions in man. Neuropsychologia, 28, $1021-1034$.

Owen AM, Roberts AC, Polkey CE, Sahakian BJ, Robbins TW (1991) Extra-dimensional versus intradimensional set shifting performance following frontal lobe excisions, temporal lobe excisions or amygdalo-hippocampectomy in man. Neuropsychologia, 29, 993-1006. 
Passingham RE (1972) Non-reversal shifts after selective prefrontal ablations in monkeys (Macaca mulatta). Neuropsychologia, 10, 41-46.

Petrides M, Milner B (1982) Deficits on subject-ordered tasks after frontal-and temporal-lobe lesions in man. Neuropsychologia, 20, 249-262.

Pillon B, Dubois B, Lhermitte F, Agid Y (1986) Heterogeneity of cognitive impairment in progressive supranuclear palsy, Parkinson's disease, and Alzheimer's disease. Neurology, Cleveland, 36, $1179-1185$.

RoBrins TW (1977) A critique of the methods available for the measurement of spontaneous motor activity. In: Handbook of Psychopharmacology, Volume 7. Edited by L. L. Iversen, S. D. Iversen and S. H. Snyder. New York: Plenum Press, pp. 37-82.

Rogers D (1986) Bradyphrenia in parkinsonism: a historical review. Psychological Medicine, 16, 257-265.

Rogers D, Lees AJ, Smith E, Trimble M, Stern GM (1987) Bradyphrenia in Parkinson's disease and psychomotor retardation in depressive illness: an experimental study. Brain, 110, 761-776.

Sagar HJ, Cohen NJ, Sullivan EV, Corkin S, Growdon JH (1988a) Remote memory function in Alzheimer's disease and Parkinson's disease. Brain, 111, 185-206.

Sagar HJ, Sullivan EV, Gabrieli JDE, Corkin S, Growdon JH (1988b) Temporal ordering and shortterm memory deficits in Parkinson's disease. Brain, 111, 525-539.

Sahakian BJ, Morris RG, Evenden Jl, Heald A, Levy R, Philpot M et al. (1988) A comparative study of visuospatial memory and learning in Alzheimer-type dementia and Parkinson's disease. Brain, $111,695-718$.

Sahakian BJ, Downes JJ, Eagger S, Evenden Jl, Levy R, Philpot MP et al. (1990) Sparing of attentional relative to mnemonic function in a subgroup of patients with dementia of the Alzheimer type. Neuropsychologia, 28, 1197-1213.

Sahgal A, Sahakian BJ, Robbins TW, Wray CJ, Lloyd S, Cook JH et al. (1991) Detection of visual memory and learning deficits in Alzheimer's disease using the Cambridge Neuropsychological Test Automated Battery. Dementia, 3, 150-158.

Saint-Cyr JA, Taylor AE, Lang AE (1988) Procedural learning and neostriatal dysfunction in man. Brain, 111, $941-959$.

Scatton B, Javoy-Agid F, Rouquier L, Dubois B, Agid Y (1983) Reduction of cortical dopamine, noradrenaline, serotonin and their metabolites in Parkinson's disease. Brain Research, Amsterdam, 275, $321-328$.

Shaluce T (1982) Specific impairments of planning. Philosophical Transactions of the Royal Society of London, B, 298, 199-209.

Starkstein SE, Preziosi TJ, Berthier ML, Bolduc PL, Mayberg HS, Robinson RG (1989) Depression and cognitive impairment in Parkinson's disease. Brain, 112, 1141-1153.

Sullivan EV, CoRKIN S, Growdon JH (1986) Verbal and nonverbal short-term memory in patients with Alzheimer's disease and in normal healthy subjects. Developmental Neuropsychology, 2, 387-400.

Taylor AE, Saint-Cyr JA, Lang AE (1986) Frontal lobe dysfunction in Parkinson's disease. Brain, $109,845-883$.

TAYLOR AE, SAINT-CYR JA, LANG AE (1987) Parkinson's disease: cognitive changes in relation to treatment response. Brain, 110, 35-51.

Watts FN, MacLeod AK, Morris L (1988) Associations between phenomenal and objective aspects of concentration problems in depressed patients. British Journal of Psychology, 79, 241-250.

Weingartner H, Burns S, Diebel R, LeWITt PA (1984) Cognitive impairment in Parkinson's disease: distinguishing between effort-demanding and automatic cognitive processes. Psychiatry Research, 11, $223-235$.

Yesavage JA, Brink TL, Rose TL, Lum O, Huang V, Adey M et al. (1982) Development and validation of a geriatric depression screening scale: a preliminary report. Journal of Psychiatric Research, 17, $37-49$. 
